\title{
The Calm before the Storm: Haemorrhagic cardiac arrest during lumbar disc surgery
}

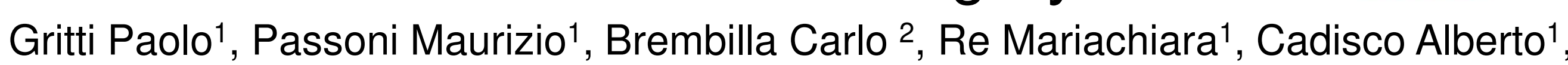 \\ Lorini Ferdinando Luca ${ }^{1}$ \\ 1 ASST Papa Giovanni XXIII ${ }^{\circ}$ Bergamo, Department of Anaesthesiology \& Intensive Care, Bergamo, Italy \\ 2 ASST Papa Giovanni XXIII ${ }^{\circ}$ Bergamo, Neurosurgery, Bergamo, Italy
}

\section{BACKGROUND}

Lumbar disc surgery is a very common surgical procedure in neurosurgery and orthopaedic surgery. Although several surgical complications could happen during the approach to the disc, hemorrhage due to vascular injuries is a rare (0.01-0.05\%) and underhanded event. ${ }^{1}$ Here we report a case of hemorrhagic cardiac arrest due to vascular injury following lumbar disc surgery.

\section{CASE REPORT}

A 53-year-old female underwent a neurosurgical procedure of lumbar disc surgery for a disc protrusion at the L5-S1 level. During the closure phase of the surgery time (13.05) The patient immediately became bradycardic with a heart rate of $35-38 / \mathrm{min}$. A dose of $0.5 \mathrm{mg}$ of atropine was administered two times but without effect. Suddenly carbon dioxide curve decrease to $10 \mathrm{mmHg}$, the plethysmography curve disappeared and non-invasive blood pressure was immeasurable. The final neurosurgery phase was interrupted and the patient was rapidly turned to the supine position. She was pulseless so an advanced cardiac life support maneuver was started. The rhythm was not shockable so adrenaline was administered in several bolus without having any perceptible pulsation. When the patient was prono-supined the abdomen was distended. Intraabdominal bleeding was suspected and vascular surgeon and radiologist were immediately alerted. A zero negative blood pack was administered immediately while (13:35) the vascular surgeon performed a laparotomy and a retroperitoneal hematoma was drained. After opening the retroperitoneal space and having evacuated the hematoma, a three $\mathrm{mm}$ of right iliac artery laceration was founded near the internal iliac artery. The laceration was repaired with monofilament $5 / 0$ and double overlook suture. The cardiac massage was protracted for fifty-five minutes. It was stopped just ten minutes after the vascular surgery reparation. Valid pulses were founded and sustained by a continuous infusion of adrenaline at $0.05 \mathrm{gamma} / \mathrm{kg}$. An overall of $18 \mathrm{mg}$ of adrenaline in single bolus of $1 \mathrm{mg}$ were administrated to the patient with $4 \mathrm{mg}$ of atropine. The patient was sent to intensive care unit with bilateral fixed mydriatic pupils ( $7 \mathrm{~mm}$ of diameter) and with a pneumothorax and edema at the neck due to the several attempts to find a central venous catheter placement in jugular and subclavian veins. The day after admission in ICU, the patient underwent a clinical evaluation which showed a rapid regaining of consciousness and motility. After that the patient was rapidly extubated and three days after she was transferred back to neurosurgical ward.

\section{DISCUSSION}

Since in 1945 Linton and White first reported vascular injury as a possible complication of disc surgery, it has been estimated that symptomatic ventral perforations occurring in $1.6-6$ per 10,000 cases. However this complication is more common than the medical community would expect. ${ }^{1,2}$ The lesions of the large pelvic vessels are described more often when working on the intervertebral space L4-L5 or L5-S1. Both because they are the most common sites of herniated discs, and because at this level the great vessels are relatively "real estate" and very close to each other (Fig 1). Consequently, the most often affected vessel is the left common iliac artery, which lies immediately in front of the fourth intervertebral disc followed by the $\mathrm{A}-\mathrm{V}$ fistula between the right common iliac artery and the left common iliac vein. Much rarer are the injuries of the inferior vena cava and the aorta, this may occur during interventions at L2-L3 and L3-L4. The vascular injury can be divided in to acute $(38 \%)$ and late types (62\%) by extension of the trauma of vessels and rapidity of blood loss. ${ }^{2}$ The acute form is mainly due to laceration of the vassels $(30 \%$ of cases) while arteriovenous fistula (67\%) or pseudoaneurysm (3\%) constitute the rest. The most dramatic context is obviously an arterial laceration. In this case you can get in a short time to haemorrhagic shock, with a mortality rate up to $80 \%$ to $100 \%$ if the problem is not promptly recognized and treated. ${ }^{2,} 3$ It is important to note that conventional advanced cardiac life support algorithms (ACLS) are not always feasible during some surgical setting. Moreover the management of intraoperative 3 cardiac arrest during neurosurgical procedures is still poorly defined. ${ }^{4}$ The position made to take the patient ( supine knee-chest position, with the abdomen free from compression) and a certain effect "self-sealing" of the anterior longitudinal ligament may cause that in the operative field does not observe a particular bleeding, despite the tearing of a great vessel. Moreover, the spread of bleeding in the retroperitoneum may be very abundant before it becomes an obvious abdominal distension, especially in "corpulent"patients Therefore, the sign of arterial lacerations usually occurs in the final stages of the intervention with all the signs of hypovolemic shock as hypotension, collapse $\mathrm{EtCO} 2$ and pallor not be explained by other causes. When in doubt, it is essential to prone-supine 4 (gear up) as soon as possible to perform an emergency laparotomy, without losing precious time in diagnostic tests a part abdominal ultrasound, if readily available. Contemporary hypovolemia should be $\mathbf{5}$ treated aggressively and all the advanced cardiac life support operations must be immediately put in practice.

\section{LEARNING POINTS}

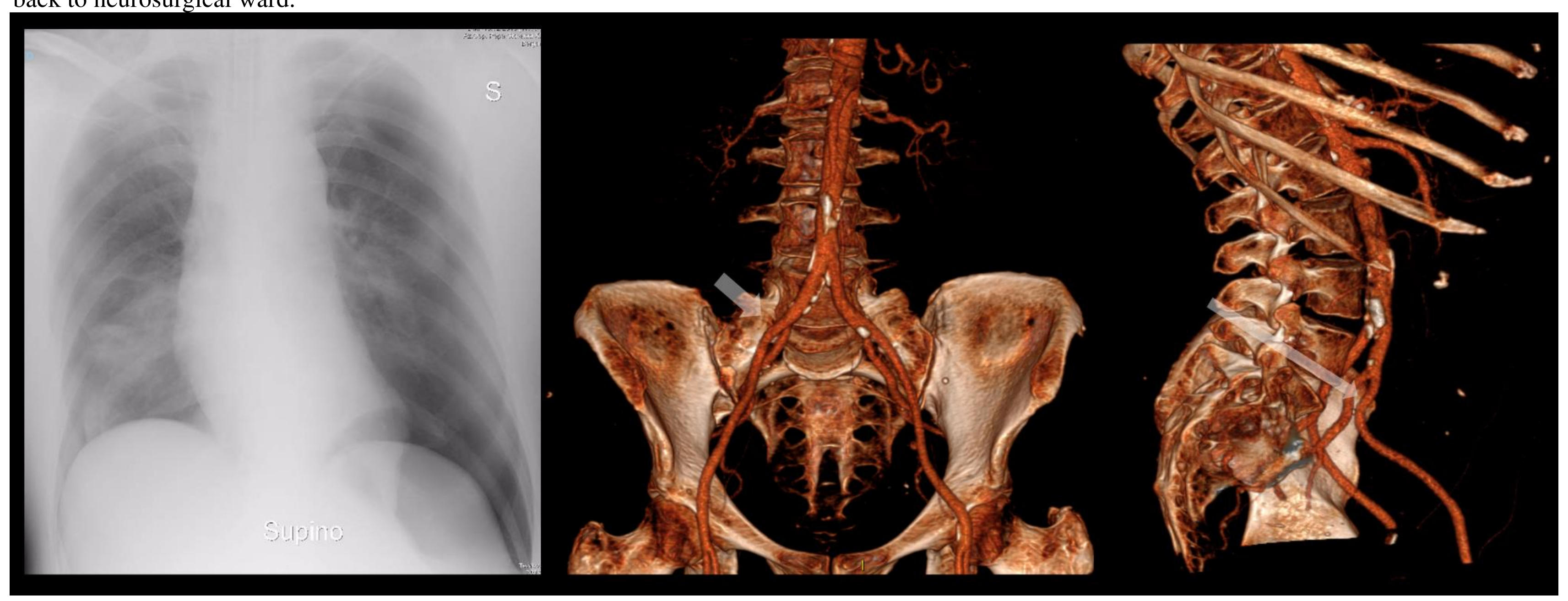

Figure $n^{\circ} 1$ On the left the bilateral pneumothorax due to the several attempts to find a central venous catheter placement in jugular and subclavian veins The lesions of the large pelvic vessels are described more often when working on the intervertebral space L4L5 or L5S1 because at this level the great vessels are relatively "real estate" and very close to each other (right side) 